Portland State University

PDXScholar

3-1-1989

\title{
Spontaneous coherent pulsations in standing-wave laser oscillators: frequency characteristics
}

Lee W. Casperson

Portland State University

Follow this and additional works at: https://pdxscholar.library.pdx.edu/ece_fac

Part of the Electrical and Computer Engineering Commons

Let us know how access to this document benefits you.

\section{Citation Details}

Lee W. Casperson, "Spontaneous coherent pulsations in standing-wave laser oscillators: frequency characteristics," J. Opt. Soc. Am. B 5, 970-978 (1988).

This Article is brought to you for free and open access. It has been accepted for inclusion in Electrical and Computer Engineering Faculty Publications and Presentations by an authorized administrator of PDXScholar. Please contact us if we can make this document more accessible: pdxscholar@pdx.edu. 


\title{
Spontaneous coherent pulsations in standing-wave laser oscillators: frequency characteristics
}

\author{
Lee W. Casperson \\ Department of Electrical Engineering, Portland State University, Portland, Oregon 97207
}

Received September 10, 1987; accepted December 7, 1987

\begin{abstract}
The output signal from a spontaneously pulsing laser must, by definition, consist of more than one frequency component. The experimental and theoretical study of the frequency components associated with such a laser can provide valuable insights into the pulsation processes. The results of an investigation into the frequency characteristics of a spontaneously pulsing standing-wave laser are reported.
\end{abstract}

\section{INTRODUCTION}

There have been numerous recent studies of the spontaneous pulsation instabilities that can sometimes occur in laser oscillators. The semiclassical instabilities exhibited by certain low-pressure gas lasers are now among the best understood, and existing models for single-mode unidirectionalring and standing-wave lasers seem to be capable of providing quantitative agreement with a wide range of experimental results. In another paper in this issue of the journal a standing-wave laser model is reported that incorporates many of the basic physical characteristics and the best available parameter values for a xenon laser. ${ }^{1}$ The time-domain predictions of that model were examined in detail and include intensity waveforms and Lamb-dip average-intensity tuning curves. Reasonable agreement with available experimental data has been obtained.

It is sometimes useful to characterize laser instabilities by means of measurements of quantities other than the instantaneous and time-averaged intensity. Measurements of the frequency content of the pulsations, for example, are relatively straightforward and can provide insight into the underlying instability dynamics. Our earliest studies of xenon-laser instabilities focused equally on the time- and frequency-domain characteristics, ${ }^{2}$ and frequency studies have also been emphasized in the experiments of Abraham et al. ${ }^{3}$ An advantage of frequency-domain measurements is that, in combination with signal averaging or synchronous detection techniques, they can be effective at extremely low signal levels. Such noise-reduction methods are obviously not applicable in time-domain measurements of aperiodic signals, and hence time measurements are not possible in low-power lasers close to threshold. Additionally, a frequency-space representation can sometimes offer a concise summary of a large amount of complicated information.

The purpose of this study is to explore the frequencydomain implications of the standing-wave laser model described in the accompanying paper. ${ }^{1}$ The frequency content of the pulsations can be represented in several different ways. We can define the instantaneous frequency as the rate of change of the phase of the laser field amplitude. This frequency varies strongly during the evolution of the individual laser pulses, and the most rapid variations tend to occur when the intensity level is lowest. The instantaneous frequency can be readily computed as an extension of the basic time-domain solutions.

It is also possible to compute the homodyne and heterodyne spectra associated with the pulsations, Experimentally, homodyne spectra are obtained by mixing a laser signal with itself at a square-law detector. Heterodyne spectra, on the other hand, are somewhat more difficult since they involve mixing the unknown optical signal with a stable reference optical signal of suitable intensity. Computationally, however, both types of spectra follow directly from the timedomain solutions.

The basic theoretical model on which this study is based is summarized briefly in Section 2, and a method for computing the instantaneous frequency is described in Section 3. Numerical results for the instantaneous frequency can be interpreted qualitatively in terms of a mode-pulling model. A series of computed homodyne spectra is given in Section 4 together with related experimental data. Heterodyne spectra are discussed in Section 5.

\section{THEORETICAL MODEL}

The general theoretical model on which this study is based is described in Ref. 1. The model was developed specifically to represent the spontaneous pulsation phenomena that had been observed in standing-wave xenon lasers, but the formulation is general enough that it should also apply to other laser types. In its normalized single-isotope gas-laser form, the model is represented by Eqs. (88)-(93) of Ref. 1, and these equations are

$$
\begin{aligned}
\frac{\partial P_{r, 2 j+1}(V, t)}{\partial t}= & -\gamma\left\{[1+(2 j+1) i V] P_{r, 2 j+1}(V, t)\right. \\
& +y P_{i, 2 j+1}(V, t)+i A_{i}(t) \\
& \left.\times\left[D_{2 j}(V, t)-D_{2 j+2}(V, t)\right]\right\}, \\
\frac{\partial P_{i, 2 j+1}(V, t)}{\partial t}= & -\gamma\left\{[1+(2 j+1) i V] P_{i, 2 j+1}(V, t)\right. \\
& -y P_{r, 2 j+1}(V, t)-i A_{r}(t) \\
& \left.\times\left[D_{2 j}(V, t)-D_{2 j+2}(V, t)\right]\right\},
\end{aligned}
$$




$$
\begin{aligned}
\frac{\partial D_{2 j}(V, t)}{\partial t}= & {\left[\lambda_{a}(V, t)-\lambda_{b}(V, t)\right] \delta_{j o} } \\
& -\left[h_{1}+(2 j) i \gamma V\right] D_{2 j}(V, t)-h_{2} M_{2 j}(V, t) \\
& -i \gamma_{1}\left\{\left[A_{r}(t) P_{i, 2 j-1}(V, t)-A_{i}(t) P_{r, 2 j-1}(V, t)\right]\right. \\
& \left.-\left[A_{r}(t) P_{i, 2 j+1}(V, t)-A_{i}(t) P_{r, 2 j+1}(V, t)\right]\right\} \\
& +\frac{\epsilon \Gamma_{a}}{2 \pi^{1 / 2}} \exp \left(-\epsilon^{2} V^{2}\right) \\
& \times \int_{-\infty}^{\infty}\left[M_{2 j}\left(V^{\prime}, t\right)+D_{2 j}\left(V^{\prime}, t\right)\right] d V^{\prime} \\
& -\frac{\epsilon \Gamma_{b}}{2 \pi^{1 / 2}} \exp \left(-\epsilon^{2} V^{2}\right) \\
& \times \int_{-\infty}^{\infty}\left[M_{2 j}\left(V^{\prime}, t\right)-D_{2 j}\left(V^{\prime}, t\right)\right] d V^{\prime}
\end{aligned}
$$

$$
\frac{\partial M_{2 j}(V, t)}{\partial t}=\left[\lambda_{a}(V, t)+\lambda_{b}(V, t)\right] \delta_{j o}-\left[h_{3}+(2 j) i \gamma V\right]
$$$$
\times M_{2 j}(V, t)-h_{4} D_{2 j}(V, t)+\frac{\epsilon \Gamma_{a}}{2 \pi^{1 / 2}}
$$$$
\times \exp \left(-\epsilon^{2} V^{2}\right) \int_{-\infty}^{\infty}\left[M_{2 j}\left(V^{\prime}, t\right)+D_{2 j}\left(V^{\prime}, t\right)\right] d V^{\prime}
$$$$
+\frac{\epsilon \Gamma_{b}}{2 \pi^{1 / 2}} \exp \left(-\epsilon^{2} V^{2}\right)
$$

$$
\begin{aligned}
& \times \int_{-\infty}^{\infty}\left[M_{2 j}\left(V^{\prime}, t\right)-D_{2 j}\left(V^{\prime}, t\right)\right] d V^{\prime}, \\
\frac{\mathrm{d} A_{r}(t)}{\mathrm{d} t}= & -\frac{1}{2 t_{c}}\left[A_{r}(t)+\delta\left(y-y_{0}\right) A_{i}(t)\right.
\end{aligned}
$$$$
\left.-\int_{-\infty}^{\infty} P_{i, 1 i}(V, t) \mathrm{d} V\right]
$$

$$
\begin{aligned}
\frac{\mathrm{d} A_{i}(t)}{\mathrm{d} t}= & -\frac{1}{2 t_{c}}\left[A_{i}(t)-\delta\left(y-y_{0}\right) A_{r}(t)\right. \\
& \left.+\int_{-\infty}^{\infty} P_{r, 1 i}(V, t) \mathrm{d} V\right] .
\end{aligned}
$$

time-dependent function can be written in terms of an amplitude and a phase, and such representations are especially useful for quasi-monochromatic signals in which slowly varying amplitude and phase variations can be isolated. The electromagnetic fields in a spontaneously pulsing laser are of this quasi-monochromatic form, and we define the instantaneous frequency as the time derivative of the total phase of the electric field. The related concepts of timedependent or instantaneous spectra have been investigated in studies of stochastic processes. .,5 $^{4}$

The normalized complex amplitude of the electric field at a point in space can be written in any of the forms

$$
\begin{aligned}
A(t)= & 1 / 2\left[A_{r}(t)+i A_{i}(t)\right] \exp (-i \omega t)+\text { c.c. } \\
= & 1 / 2\left[A_{r}^{2}(t)+A^{2}(t)\right]^{1 / 2} \\
& \times \exp \left\{-i \omega t+i \tan ^{-1}\left[A_{i}(t) / A_{r}(t)\right]\right\}+\text { c.c. } \\
= & {\left[A_{r}^{2}(t)+A_{i}^{2}(t)\right]^{1 / 2} \cos \left\{\omega t-\tan ^{-1}\left[A_{i}(t) / A_{r}(t)\right]\right\} } \\
= & A_{r}(t) \cos (\omega t)+A_{i}(t) \sin (\omega t),
\end{aligned}
$$

where $A_{r}(t)$ and $A_{i}(t)$ are obtained as numerical solutions of Eqs. (1)-(6). Thus the total phase of the field can be written as

$$
\phi(t)=\omega t-\tan ^{-1}\left[A_{i}(t) / A_{r}(t)\right] .
$$

The time-dependent frequency is

$$
\begin{aligned}
\omega(t) & =\frac{\mathrm{d} \phi(t)}{\mathrm{d} t} \\
& =\omega-\frac{\mathrm{d}}{\mathrm{d} t}\left\{\tan ^{-1}\left[\frac{A_{i}(t)}{A_{r}(t)}\right]\right\} .
\end{aligned}
$$

In normalized units this frequency is

$$
y(t)=y-\frac{1}{\gamma} \frac{\mathrm{d}}{\mathrm{d} t}\left\{\tan ^{-1}\left[\frac{A_{i}(t)}{A_{r}(t)}\right]\right\} .
$$

The instantaneous frequency can be calculated directly from Eq. (10) with the computed values of $A_{i}(t)$ and $A_{r}(t)$, provided that one is careful with the abrupt changes between $-\pi / 2$ and $+\pi / 2$ in the principal values computed for $\tan ^{-1}$. Examples of the time-dependent frequency for a threshold parameter of $r=1.4$ and various positive frequency detunings are shown in Fig. 1. The frequencies are plotted immediately below the corresponding intensity waveforms. At line center with $r=1.4$, the intensity is in the form of quasi-periodic pulsation bursts (as shown in Fig. 1 of Ref. 1). With detuning the echoes evolve to intermediate pulses that at the detuning $\Delta \nu=7.5 \Delta \nu_{h}$ have become indistinguishable from the principal pulses. The frequency characteristics also evolve with detuning. The lower trace in Fig. 1(a) shows the time-dependent normalized frequency shift away from the nondispersed cavity frequency. If it were not for the dispersion due to the laser transition, the lasing frequency would be along the line of dots marking the center of the plot. The fact that the frequency is usually below the center of the plot is an indication that the dispersion has pulled the mode closer to line center.

The mode-pulling interpretation can also be stated more quantitatively. The nondispersed lasing frequency for Fig.

The frequency information carried by the pulses can be represented in several different ways. One possibility is to introduce the concept of instantaneous frequency. Any 

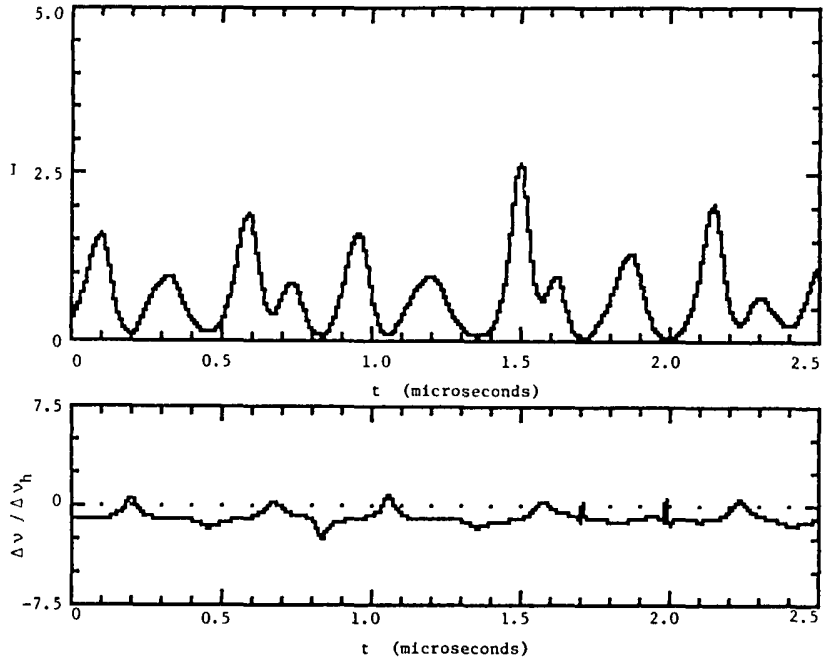

(a)
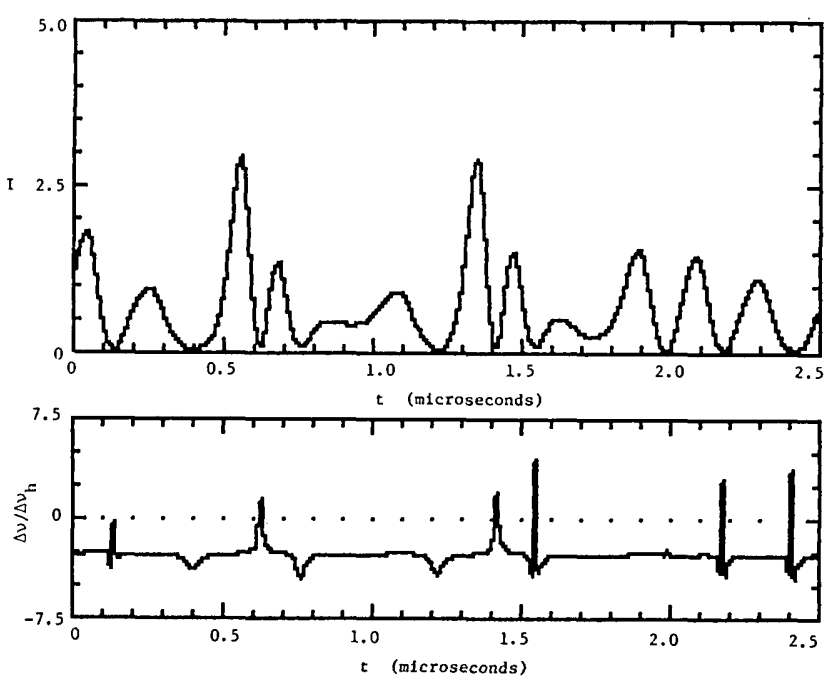

(b)

1 (a) is detuned up from line center by the amount $\Delta \nu=$ $2.5 \Delta \nu_{h}$. But the lower curve in Fig. 1(a) shows that the actual lasing frequency is pulled back down from the detuning offset by an average of approximately $\Delta \nu=1 \Delta \nu_{h}$. Thus the actual laser frequency detuning is the fraction $(2.5-1)$ / $2.5=0.60$ of the nondispersed detuning. This result includes saturation effects and hence indicates somewhat less mode pulling than would be predicted by conventional linear mode-pulling theory for tuning near line center. According to that theory, the detuning would have been reduced by the factor $(1+\beta)^{-1}$, where the dimensionless dispersion parameter $\beta$ for a uniform resonator in the Doppler inhomogeneous broadening limit is defined as ${ }^{6}$

$$
\beta=\frac{\operatorname{cg}(\log 2)^{1 / 2}}{\pi^{3 / 2} \Delta \nu_{d \mathrm{~d}}} .
$$

If the amplifier gain rate $c g$ is identified with the product of the threshold parameter (1.4 in this example) and the cavity loss rate $t_{c}{ }^{-1}$, then the dispersion parameter for the xenon laser has the numerical value $\beta=1.4 \times 10^{9}(\log 2)^{1 / 2} /\left(\pi^{3 / 2} 110\right.$ $\left.\times 10^{6}\right)=1.9$. Thus this simplified model would imply a
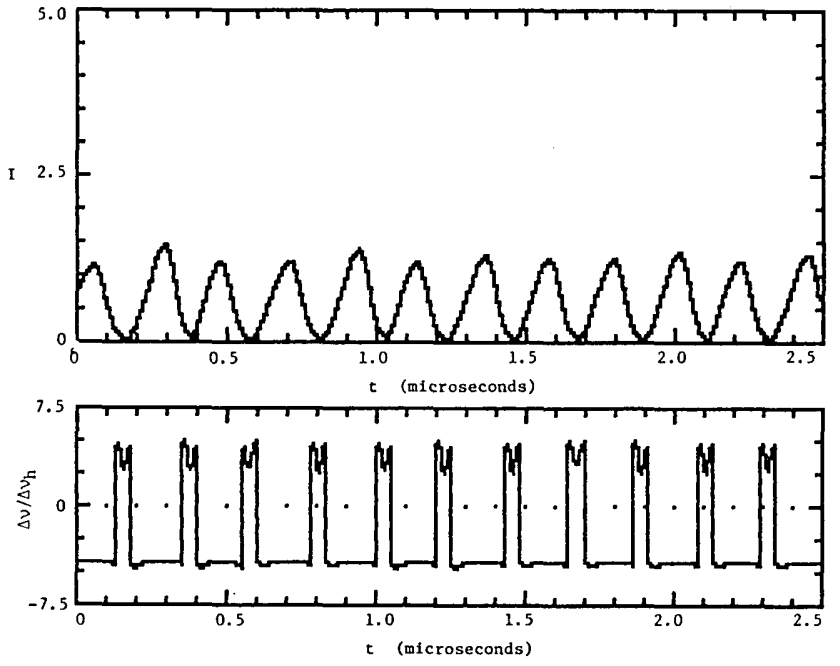

(c)

Fig. 1. Theoretical spontaneous pulsation waveforms for a xenon laser with a threshold parameter of $r=1.4$ and detunings of (a) $\Delta \nu=$ $2.5 \Delta \nu_{h}$, (b) $\Delta \nu=5.0 \Delta \nu_{h}$, and (c) $\Delta \nu=7.5 \Delta \nu_{h}$. Immediately below each intensity waveform is the corresponding normalized instantaneous frequency as referenced to the nondispersed laser frequency. Thus in the lower trace of (a), for example, the average frequency is found to be pulled about $\Delta \nu_{h}$ closer to line center than the nondispersed detuning of $2.5 \Delta \nu_{h}$.

detuning reduction by the factor $(1+1.9)^{-1}=0.34$ rather than 0.60 , as indicated by the more rigorous numerical solutions.

Some of the details of the time-dependent frequency waveform in Fig. 1(a) are also of interest. In particular, the frequency is found to depart most significantly from its average value when the intensity is near a minimum, and extremely rapid frequency changes are found to occur when the intensity is very close to zero. It is not surprising that the frequency should be able to change more quickly when the intensity is low since, under that condition, there are fewer photons present to maintain any existing frequency, and at zero intensity the frequency is, of course, entirely undefined. As shown in Figs. 1(b) and 1(c), similar behavior is obtained with other values of detuning. There is, however, a strengthening of the mode pulling as one considers spectral regions away from the Lamb dip at line center. Thus the frequency offset in Fig. 1(b) is approximately 0.5 of its nondispersed value. In Fig. 1(c) this factor is only about 0.4 , which is close to the simplified mode-pulling prediction mentioned above. Interestingly, with the larger detuning of 
Fig. 1(c) the laser mode spends most of its low-intensity time at a positive offset from the nondispersed cavity frequency.

\section{HOMODYNE SPECTRA}

Instead of displaying the instantaneous frequency, it is often more useful to examine the frequency content by means of the homodyne or heterodyne spectra. These spectra can be obtained from optical mixing experiments by using a conventional light detector. It has been shown by Forrester and others that the current in many types of photodetectors can be regarded as resulting from a squaring and then an averaging (low-pass filtering) of the incident electric field. ${ }^{7-9}$ In some respects a homodyne frequency spectrum can be considered a special case of a heterodyne spectrum, and hence we begin by considering a hypothetical heterodyne-frequency-spectrum measurement in which the unknown spectrum is mixed at the detector with a monochromatic optical reference signal (local oscillator) of frequency $\omega_{r}$ and real amplitude $C$. Thus, with Eq. (7), the total electric field incident upon the detector is

$$
\begin{aligned}
A_{\text {total }}(t)=1 / 2\left[A_{r}(t)+\right. & \left.i A_{i}(t)\right] \exp (-i \omega t) \\
& +1 / 2 C \exp \left(-i \omega_{r} t\right)+\text { c.c. }
\end{aligned}
$$

The square of this field amplitude can be written as
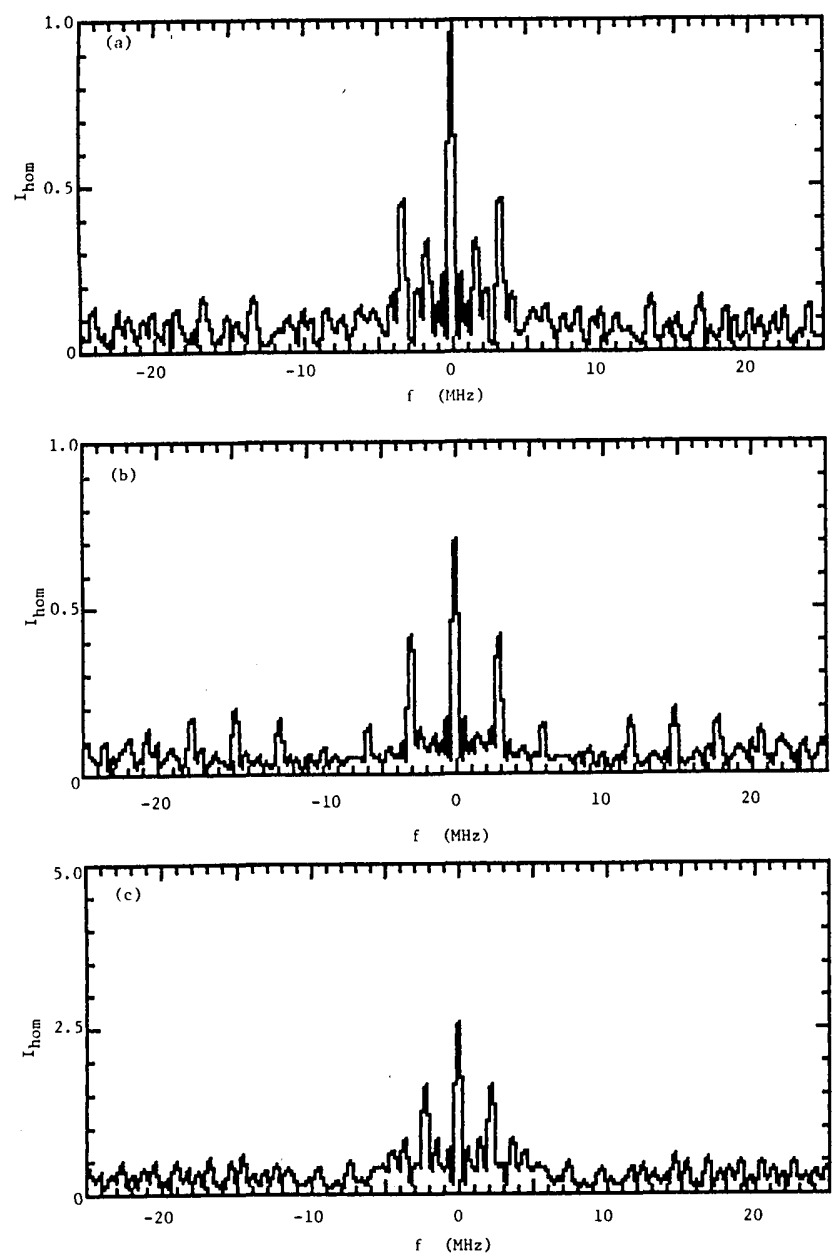

$$
\begin{aligned}
A_{\text {total }}^{2}(t)= & 1 / 2\left[A_{r}^{2}(t)+A_{i}^{2}(t)+C^{2}\right]+\left[A_{r}^{2}(t)+A_{i}^{2}(t)\right]^{1 / 2} C \\
& \times \cos \left\{\left(\omega-\omega_{r}\right) t-\tan ^{-1}\left[A_{i}(t) / A_{r}(t)\right]\right\} \\
& +1 / 2\left[A_{r}^{2}(t)+A_{i}^{2}(t)\right] \cos \left\{2 \omega t-2 \tan ^{-1}\left[A_{i}(t) / A_{r}(t)\right]\right\} \\
& +1 / 2 C^{2} \cos \left(2 \omega_{r} t\right)+\left[A_{r}^{2}(t)+A_{i}^{2}(t)\right]^{1 / 2} C \\
& \times \cos \left\{\left(\omega+\omega_{r}\right) t-\tan ^{-1}\left[A_{i}(t) / A_{r}(t)\right]\right\}
\end{aligned}
$$

This result may now be averaged over a time that is long compared with an optical cycle because most detectors could not respond to the instantaneous electric field of an optical frequency signal. If the detector is considered to be fast compared with any time associated with the pulsations or with the time $\left(\omega-\omega_{r}\right)^{-1}$, then Eq. (13) suggests that the detector current would be proportional to

$$
\begin{gathered}
i_{d}(t)=1 / 2\left[A_{r}^{2}(t)+A_{i}^{2}(t)+C^{2}\right]+\left[A_{r}{ }^{2}(t)+A_{i}{ }^{2}(t)\right]^{1 / 2} C \\
\times \cos \left\{\left(\omega-\omega_{r}\right) t-\tan ^{-1}\left[A_{i}(t) / A_{r}(t)\right]\right\} .
\end{gathered}
$$

Most of the laser instability measurements that we might visualize can be interpreted as special cases of Eq. (14). In the absence of a reference signal $(C=0)$ this result predicts a photocurrent proportional to the intensity, and we will refer to this as the homodyne current
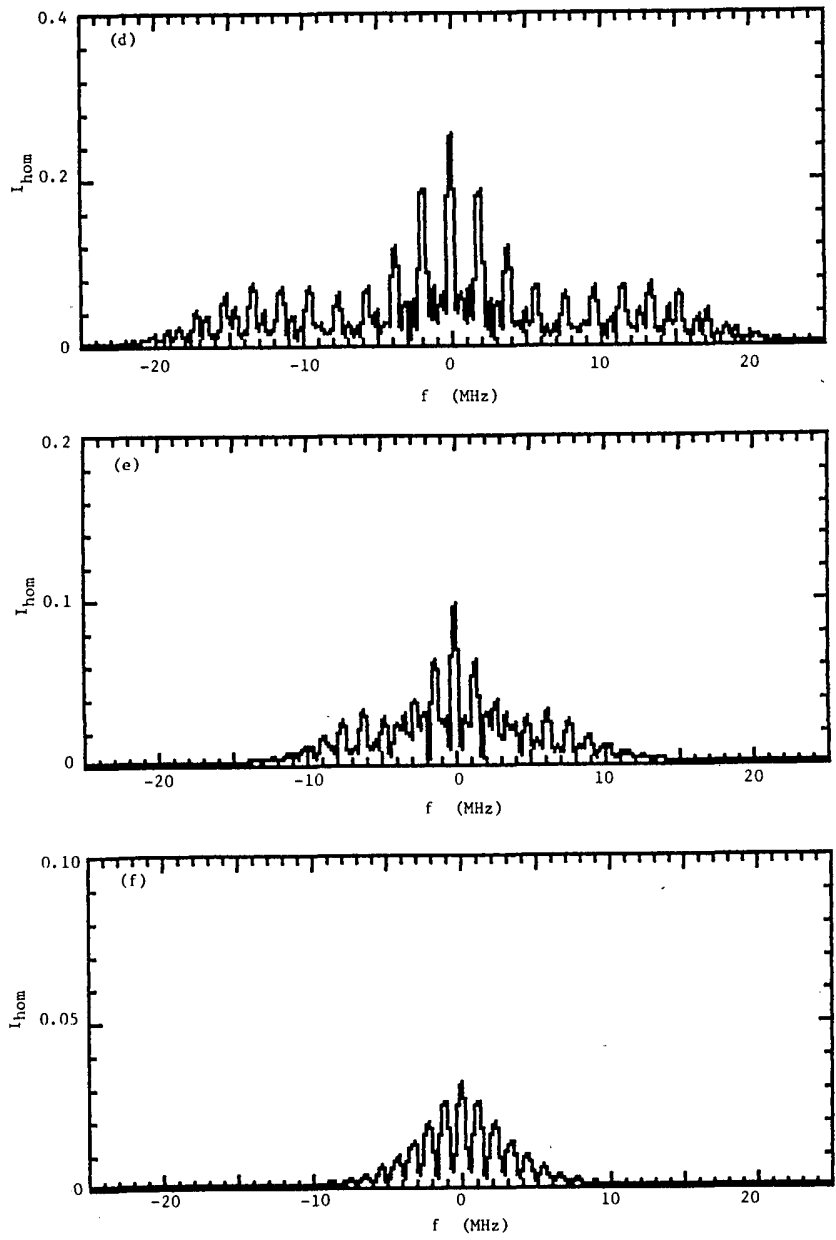

Fig. 2. Theoretical homodyne spectra corresponding to the intensity waveforms in Fig. 1 of Ref. 1 . The threshold parameters for the individual spectra are (a) $r=2.0$, (b) $r=1.8$, (c) $r=1.6$, (d) $r=1.4$, (e) $r=1.2$, and (f) $r=1.1$. 


$$
i_{d, \text { hom }}(t)=1 / 2\left[A_{r}^{2}(t)+A_{i}^{2}(t)\right] .
$$

A double-detector experiment in which both detectors receive an incident laser signal and one of them also receives a fixed-frequency reference signal would, with Eqs. (14) and (15), provide real-time experimental access to the instantaneous frequency defined in Eq. (10). Using the intensity $A_{r}{ }^{2}(t)+A_{i}{ }^{2}(t)$ and the ratio $A_{i}(t) / A_{r}(t)$, we could also obtain real-time values for the individual amplitude components $A_{r}(t)$ and $A_{i}(t)$.

Our main interest in this section is in homodyne measurements that might be based on the previous results. For a homodyne calculation the reference signal is absent, and the detector signal of Eq. (15) is fed directly into a rf spectrum analyzer. The resulting spectrum is related to the field amplitudes by

$$
\begin{aligned}
I_{\text {hom }}\left(\omega_{s}\right)= & \left\{\iint_{-\infty}^{\infty} 1 / 2\left[A_{r}{ }^{2}(t)+A_{i}{ }^{2}(t)\right] \cos \left(\omega_{s} t\right) \mathrm{d} t\right\}^{2} \\
& \left.+\left\{\int_{-\infty}^{\infty} 1 / 2\left[A_{r}{ }^{2}(t)+A_{i}{ }^{2}(t)\right] \sin \left(\omega_{s} t\right) \mathrm{d} t\right\}^{2}\right)^{1 / 2},
\end{aligned}
$$
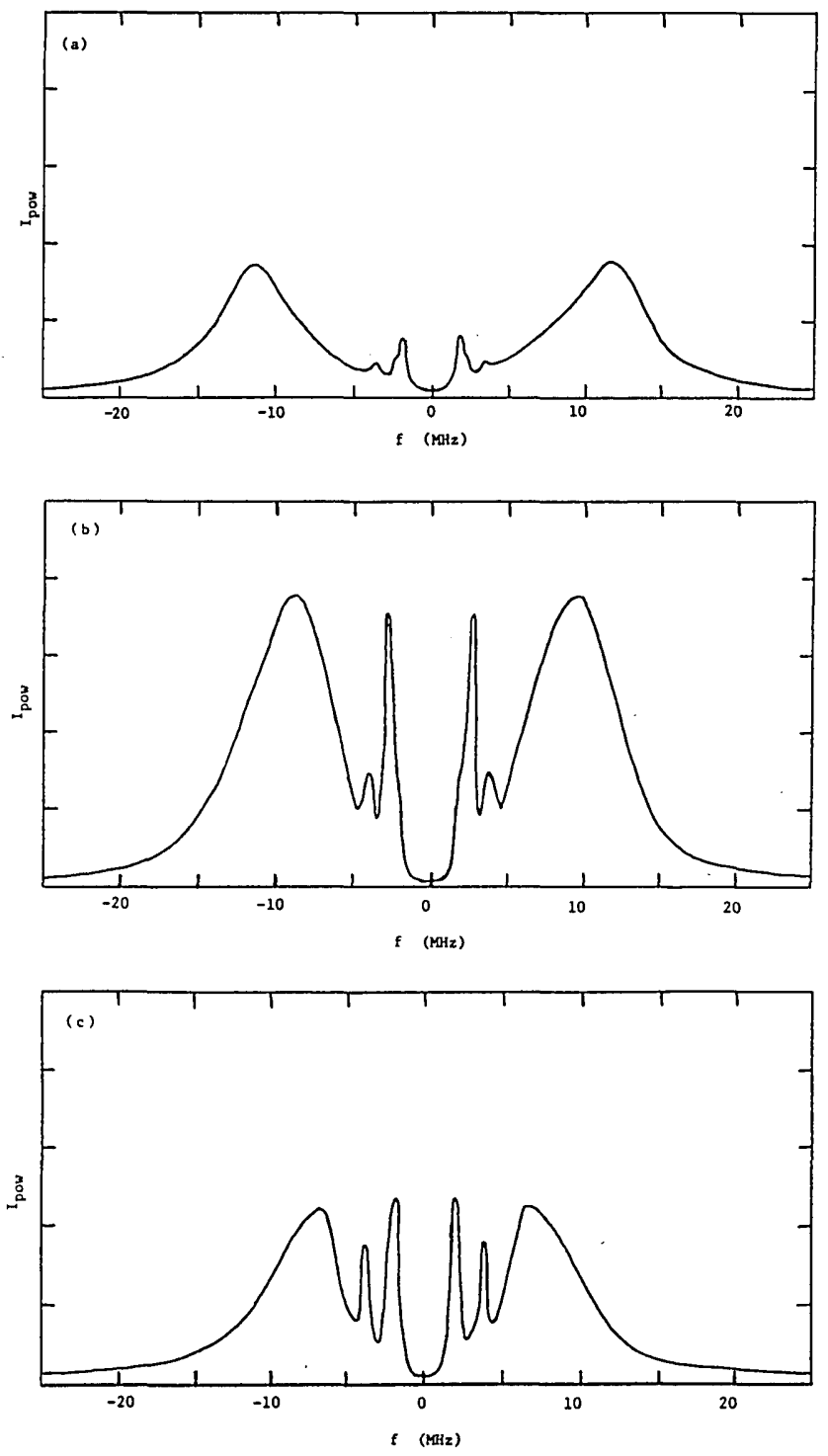

Fig. 3. Experimental homodyne spectra of the output from a low-pressure xenon laser at discharge currents of (a) $120 \mathrm{~mA}(r \approx 5.1),(\mathrm{b}) 100 \mathrm{~mA}$ $(r \approx 4.2)$, (c) $80 \mathrm{~mA}(r \approx 3.4)$, (d) $60 \mathrm{~mA}(r \approx 2.5)$, (e) $50 \mathrm{~mA}(r \approx 2.1)$, and (f) $40 \mathrm{~mA}(r \approx 1.7)$. where $\omega_{s}$ represents the scanned frequency of the analyzer and the normalization has been chosen arbitrarily. The homodyne power spectrum is the square of the result given in Eq. (16), but the power spectrum may be harder to interpret for wide variations in the intensities of the various spectral components.

As examples of the application of Eq. (16), a set of homodyne spectra is shown in Fig. 2 for a low-pressure xenon laser tuned to line center and various values of the threshold parameter. These spectra should be considered in conjunction with the corresponding time-domain intensity plots given in Fig. 1 of Ref. 1. The apparently chaotic irregularities in the pulsation waveforms lead to the broad backgrounds observable in some of the homodyne spectra. At high-excitation levels, the sharply defined frequency components may be identified with the repetition frequency of the pulsation bursts, and the shoulders on the spectra result from the high-frequency ringing within the bursts. At lower excitation the ringing and the spectral shoulders have vanished.

For comparison a set of experimental homodyne power spectra are shown in Fig. 3 for various values of the discharge
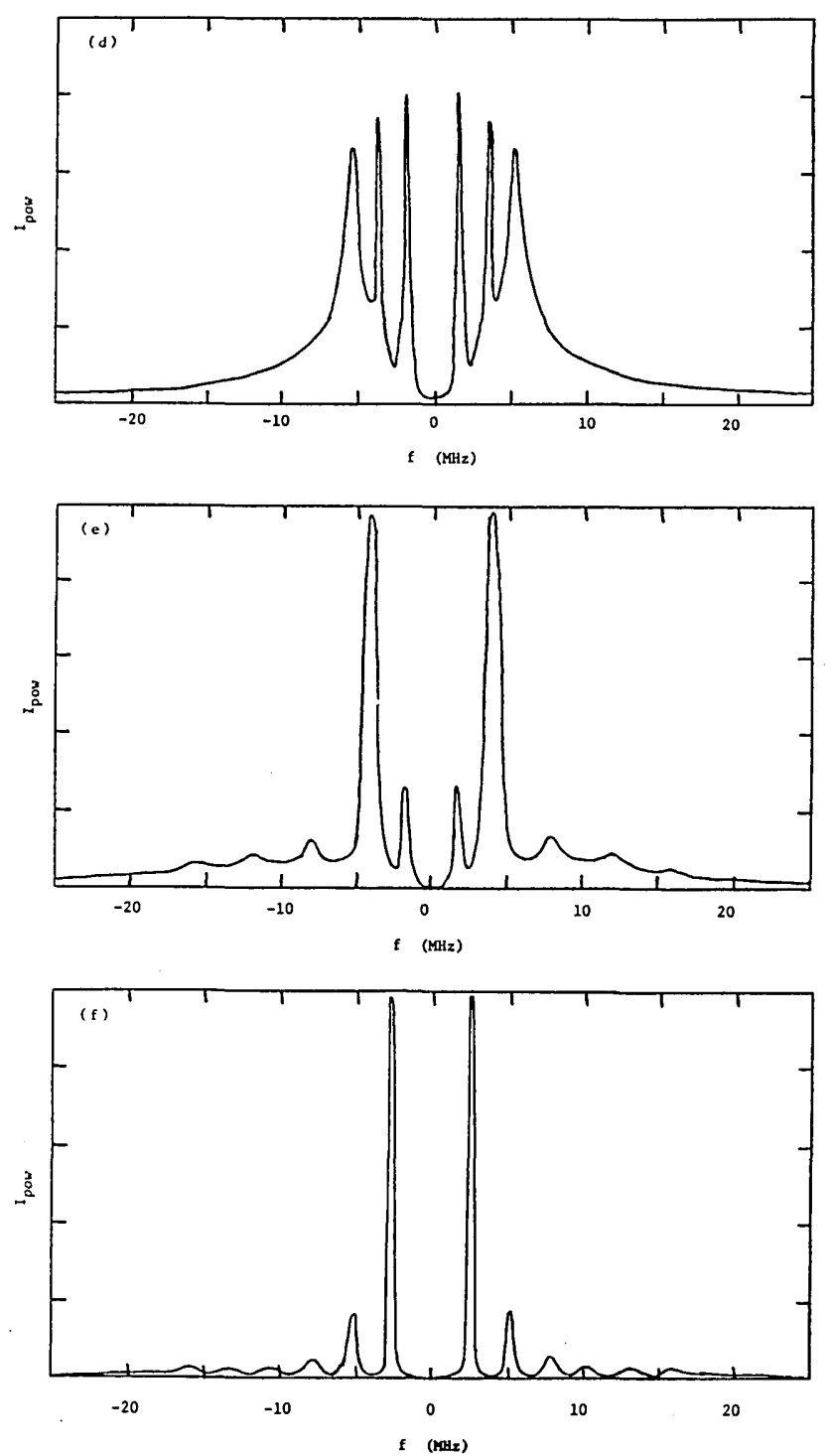

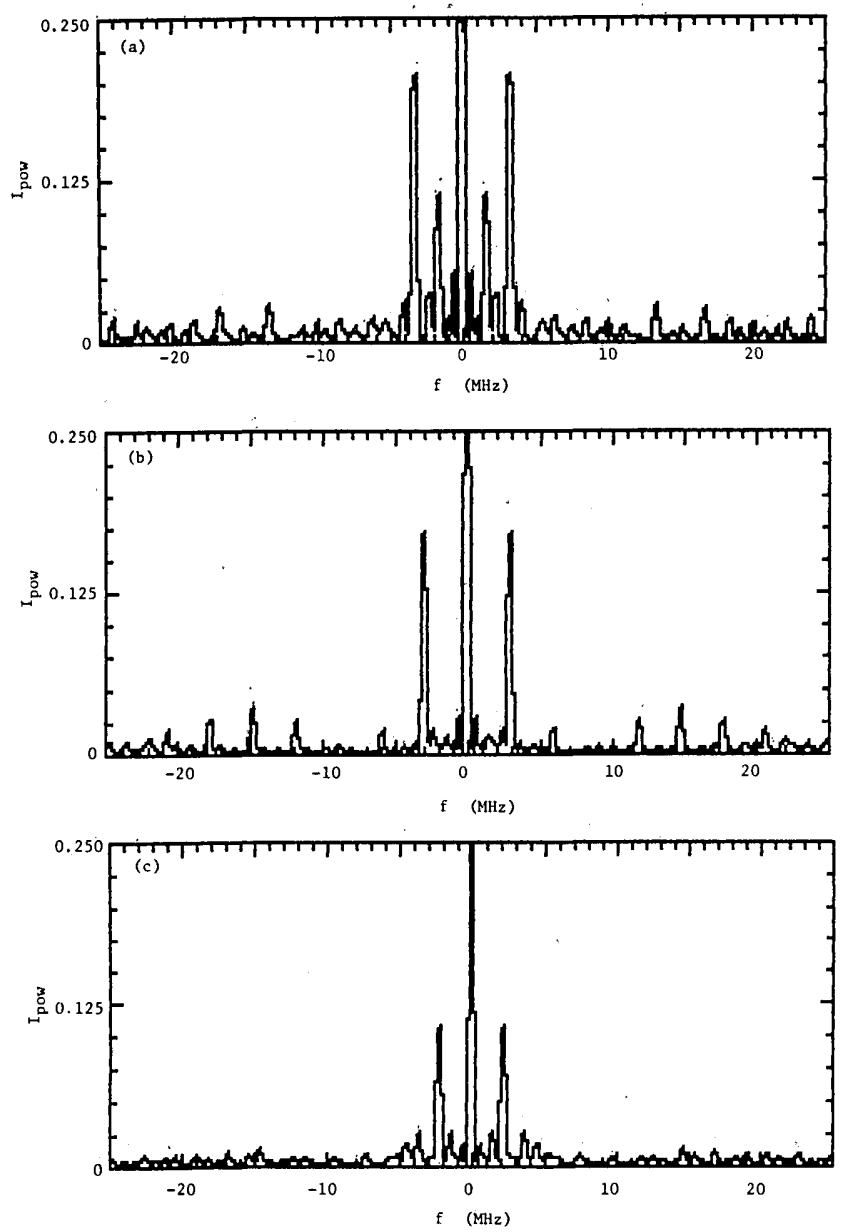

Fig. 4. Theoretical power spectra corresponding to a threshold parameter of (a) $r=2.0$ [Fig. 2(a)], (b) $r=1.8$ [Fig. 2(b)], and (c) $r=$ 1.6 [Fig. 2(c)].

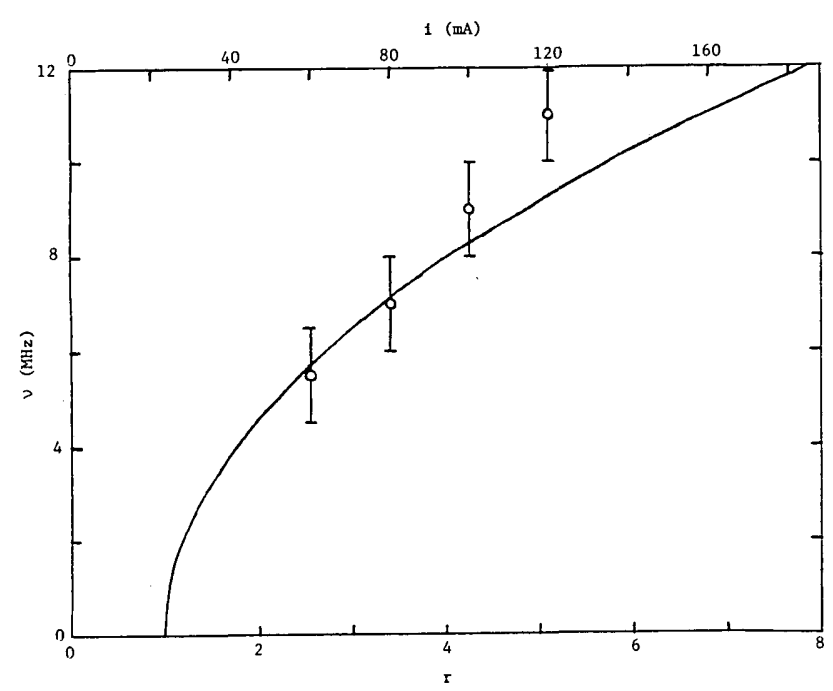

Fig. 5. Pulsation frequency versus threshold parameter. The experimental points represent the peaks of the broadband spectra shown in Fig. 3, and the solid line is the rate-equation formula given in Eq. (17). current (threshold parameter). The central zero-frequency component is not displayed by the spectrum analyzer. It is evident that for low values of the discharge current the pulsations are highly periodic, with several harmonics of the fundamental frequency in evidence. At higher current levels the broadband chaotic behavior becomes dominant, and the discrete spectral components become relatively insignificant. To yield a more direct comparison with theory, the theoretical power spectra corresponding to Figs. 2(a)-2(c) are displayed in Fig. 4. The experimental spectrum displayed in Fig. 3(f) corresponds to a threshold parameter of approximately $r=1.7$ and hence is between the predicted results in Figs. 4(b) and 4(c). Alternating pulsation bursts are suggested by the inner frequency components of the homodyne power spectrum in Fig. 3(e), and this result is similar to the corresponding theoretical data in Fig. 4(a).

It has long been known that the pulsation frequencies observed in a spontaneously pulsing xenon laser can be at least qualitatively represented by a rate-equation model. ${ }^{2}$ The only difficulty with the rate-equation model is, of course, that it predicts that the pulsations should be damped. It is perhaps worth noting that the broad spectral continuum that becomes increasingly dominant for the larger threshold parameters in Fig. 3 is also reminiscent of a rate-equation model driven by a broadband noise source. This observation can be made quantitative by plotting the frequencies of the broadband peaks in Figs. 3(a)-3(d) against the corresponding threshold parameter values. This experimental plot is given in Fig. 5 together with a theoretical plot of the rate-equation result

$$
\nu=\frac{1}{2 \pi}\left[\frac{r-1}{\tau t_{c}}-\left(\frac{r}{2 \tau}\right)^{2}\right]^{1 / 2} .
$$

The values of $\tau$ and $t_{c}$ used in plotting Eq. (17) are the same as those used in the semiclassical model described above, and thus there are no adjustable parameters.

The agreement between the experimental pulsation spectra and a noise-driven rate-equation model suggests that for operation far above threshold the semiclassical model for an inhomogeneously broadened laser may be interpretable in two almost distinct parts. One part would be primarily a generator of chaotic broadband noise, and the other part would act as a set of rate equations responding to the chaotic noise input. It would not be surprising if the field equations, which produce the usual measurable output, could be reduced to some simpler rate-equation form since even the time derivatives in the field equations are unnecessary for the chaotic oscillation mechanism. ${ }^{10}$

\section{HETERODYNE SPECTRA}

For a consideration of heterodyne detection, it is convenient to write Eq. (14) in the form

$$
\begin{aligned}
i_{d}(t)= & \frac{1}{2}\left[A_{r}{ }^{2}(t)+A_{i}{ }^{2}(t)+C^{2}\right]+C \\
& \times\left\{A_{r}(t) \cos \left[\left(\omega-\omega_{r}\right) t\right]+A_{i}(t) \sin \left[\left(\omega-\omega_{r}\right) t\right]\right\} .
\end{aligned}
$$

To obtain unambiguous results we require either that the intensity of the reference local oscillator must be much larger than the signal intensity $\left[C^{2} \gg A_{r}{ }^{2}(t)+A_{i}{ }^{2}(t)\right]$ or that the inverse of the frequency offset $\left(\omega-\omega_{r}\right)^{-1}$ must be small compared with any times associated with the intensity pulsations. Otherwise, the first bracketed terms on the right- 

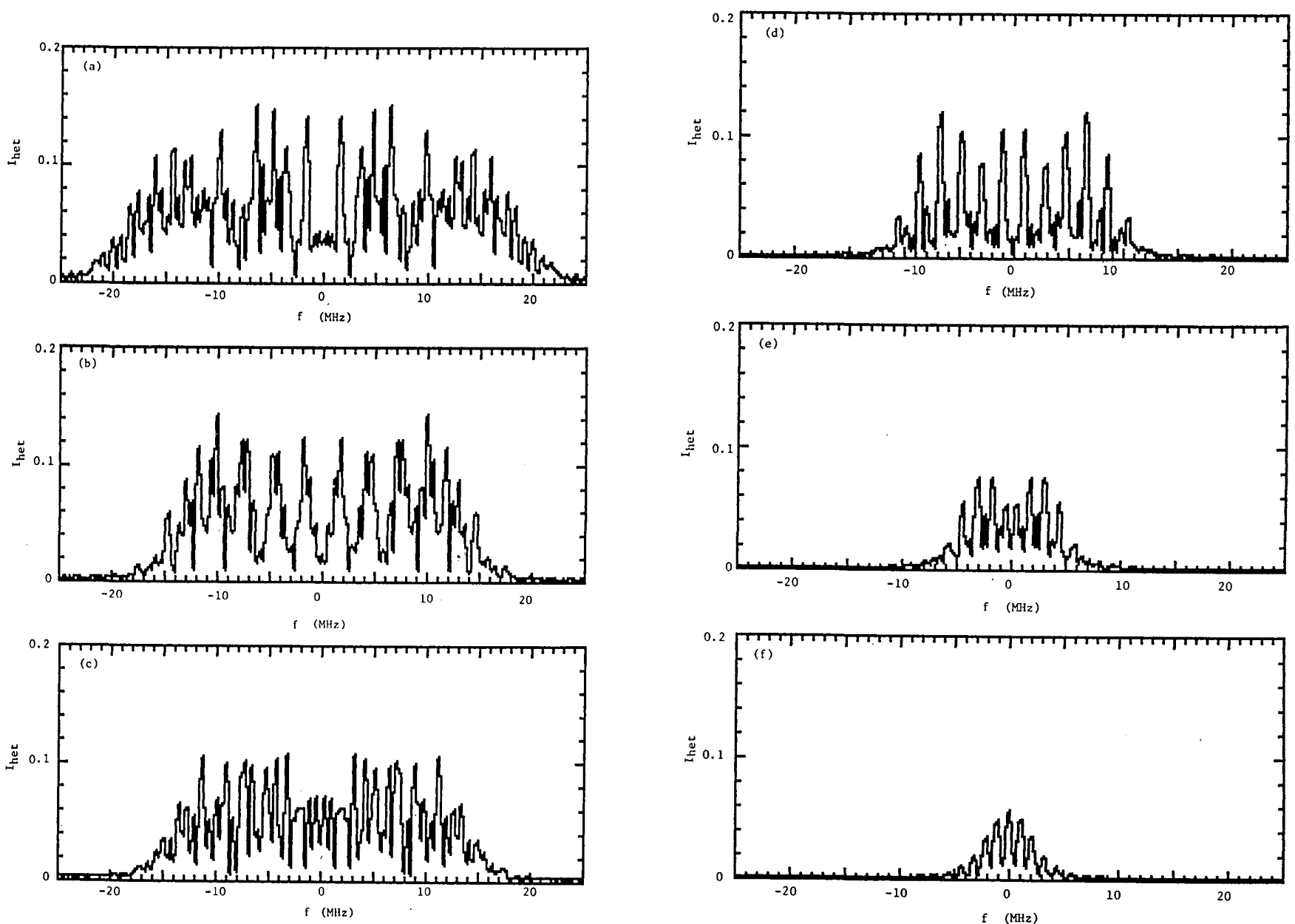

Fig. 6. Theoretical heterodyne spectra corresponding to the intensity waveforms in Fig. 1 of Ref. 1 . The threshold parameters for the individual spectra are (a) $r=2.0$, (b) $r=1.8$, (c) $r=1.6$, (d) $r=1.4$, (e) $r=1.2$, and (f) $r=1.1$.
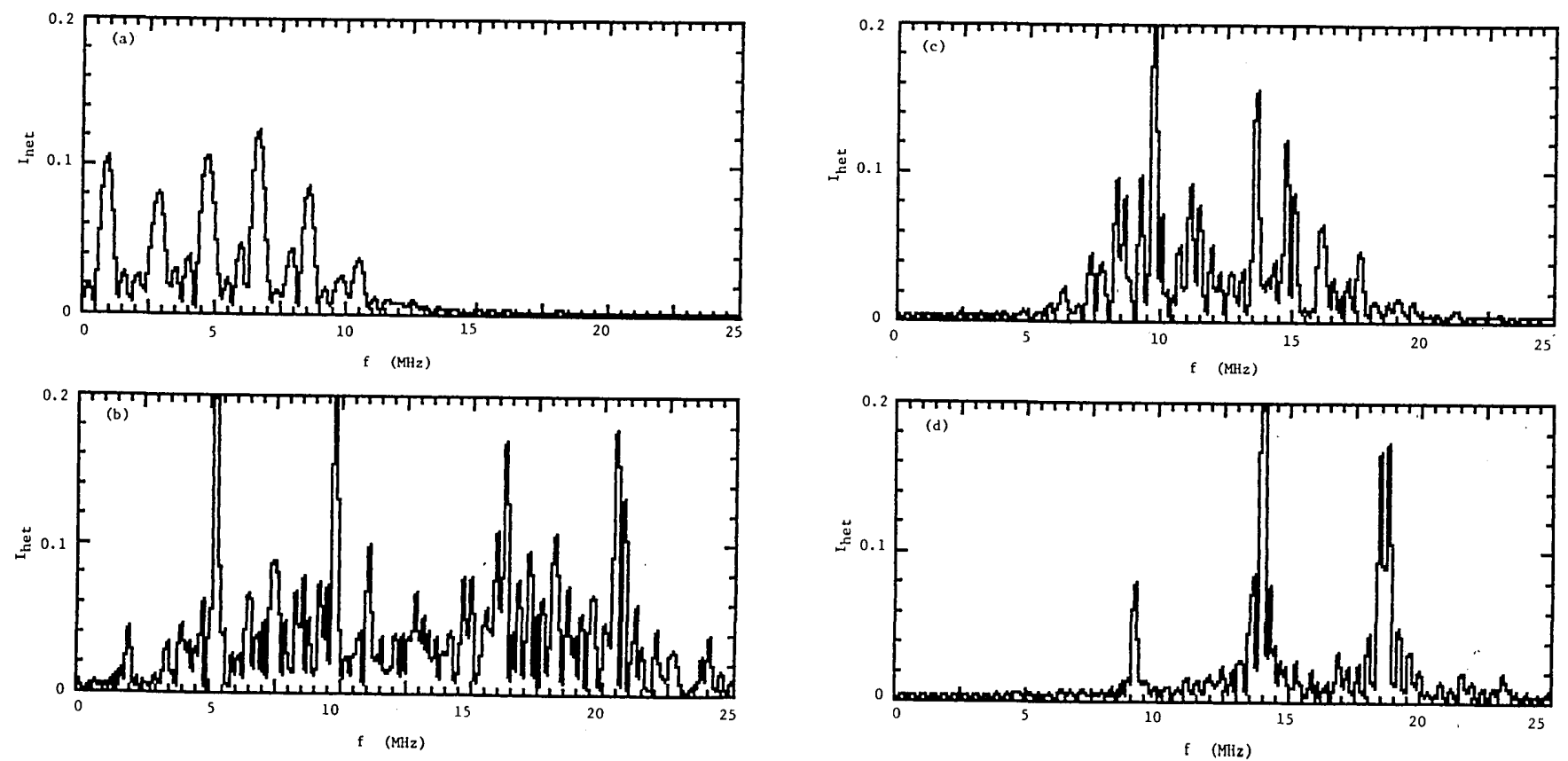

Fig. 7. Theoretical heterodyne spectra corresponding to the intensity waveforms in Fig. 1. The detunings are (a) $\Delta \nu=0 \Delta \nu_{h}$, (b) $\Delta \nu=2.5 \Delta \nu_{h}$, (c) $\Delta \nu=5 \Delta \nu_{h}$, and (d) $\Delta \nu=7.5 \Delta \nu_{h}$. 
hand side of Eq. (18) would inevitably obscure the true heterodyne results. After the first terms are eliminated by high-pass filtering, the heterodyne current can be defined as

$$
i_{d, \text { het }}(t)=C\left\{A_{r}(t) \cos \left[\left(\omega-\omega_{r}\right) t\right]+A_{i}(t) \sin \left[\left(\omega-\omega_{r}\right) t\right]\right\} \text {. }
$$

Except for the downshift of the carrier frequency, this current is directly proportional to the field amplitude given in Eq. (7). If the reference amplitude $C$ is large, then the heterodyne current can be much larger than the homodyne current given in Eq. (15), and this current enhancement can be important in considerations of signal-to-noise ratios.

There are several ways in which the actual heterodyne measurements could be performed. One possibility would be simply to pass the detector current given in Eq. (19) through a narrow-pass filter and then to display the rectified output as a function of the slowly tuned reference oscillator frequency. More commonly, however, the optical reference frequency is held constant, and the detector current given in Eq. (19) is fed directly to a conventional rf spectrum analyzer. Thus the heterodyne spectrum would be proportional to

$$
\begin{aligned}
I_{\mathrm{het}}\left(\omega_{s}\right)= & {\left[\left(\int _ { - \infty } ^ { \infty } \left\{A_{r}(t) \cos \left[\left(\omega-\omega_{r}\right) t\right]\right.\right.\right.} \\
& \left.\left.+A_{i}(t) \sin \left[\left(\omega-\omega_{r}\right) t\right]\right\} \cos \left(\omega_{s} t\right) \mathrm{d} t\right)^{2} \\
& +\left(\int _ { - \infty } ^ { \infty } \left\{A_{r}(t) \cos \left[\left(\omega-\omega_{r}\right) t\right]\right.\right. \\
& \left.\left.\left.+A_{i}(t) \sin \left[\left(\omega-\omega_{r}\right) t\right]\right\} \sin \left(\omega_{s} t\right) \mathrm{d} t\right)^{2}\right]^{1 / 2}
\end{aligned}
$$

This result may be expanded by using standard formulas for the products of trigonometric functions, and we obtain

$$
\begin{aligned}
I_{\text {het }}\left(\omega_{s}\right)= & {\left[\int _ { - \infty } ^ { \infty } \left\{\frac{A_{r}(t)}{2} \cos \left[\left(\omega_{s}+\omega-\omega_{r}\right) t\right]\right.\right.} \\
& +\frac{A_{r}(t)}{2} \cos \left[\left(\omega_{s}-\omega+\omega_{r}\right) t\right] \\
& +\frac{A_{i}(t)}{2} \sin \left[\left(\omega_{s}+\omega-\omega_{r}\right) t\right] \\
& \left.\left.-\frac{A_{i}(t)}{2} \sin \left[\left(\omega_{s}-\omega+\omega_{r}\right) t\right]\right\} \mathrm{d} t\right)^{2} \\
& +\left(\int _ { - \infty } ^ { \infty } \left\{\frac{A_{r}(t)}{2} \sin \left[\left(\omega_{s}+\omega-\omega_{r}\right) t\right]\right.\right. \\
& +\frac{A_{r}(t)}{2} \sin \left[\left(\omega_{s}-\omega+\omega_{r}\right) t\right] \\
& +\frac{A_{i}(t)}{2} \cos \left[\left(\omega_{s}+\omega-\omega_{r}\right) t\right] \\
& \left.\left.\left.-\frac{A_{i}(t)}{2} \cos \left[\left(\omega_{s}-\omega+\omega_{r}\right) t\right]\right\} \mathrm{d} t\right)^{2}\right]^{1 / 2}
\end{aligned}
$$

The spectral information of interest can be obtained by considering only frequencies close to $\omega_{s} \approx \omega-\omega_{r}$ and by introducing the new frequency $\omega_{s}^{\prime}=\omega_{s}+\omega_{r}$. Thus the significant parts of Eq. (21) can be written more compactly as

$$
\begin{aligned}
I_{\text {het }}\left(\omega_{s}^{\prime}\right)= & {\left[\left(\int _ { - \infty } ^ { \infty } \left\{\frac{A_{r}(t)}{2} \cos \left[\left(\omega_{s}^{\prime}-\omega\right) t\right]\right.\right.\right.} \\
& \left.\left.-\frac{A_{i}(t)}{2} \sin \left[\left(\omega_{s}^{\prime}-\omega\right) t\right]\right\} \mathrm{d} t\right)^{2} \\
& +\left(\int _ { - \infty } ^ { \infty } \left\{\frac{A_{r}(t)}{2} \sin \left[\left(\omega_{s}^{\prime}-\omega\right) t\right]\right.\right. \\
& \left.\left.\left.-\frac{A_{i}(t)}{2} \cos \left[\left(\omega_{s}^{\prime}-\omega\right) t\right]\right\} \mathrm{d} t\right)^{2}\right]^{1 / 2} .
\end{aligned}
$$

Examples of the application of Eq. (22) are shown in Fig. 6 for a xenon laser tuned to line center and various values of the threshold parameter. The corresponding time-domain intensity plots are given in Fig. 1 of Ref. 1, and the corresponding homodyne spectra plots are in Fig. 2 of this paper. Again, the broad background spectra observed at high-excitation levels become dominated by sharp line spectra at the pulsation harmonics when the excitation level is reduced. For operation close to threshold (e.g., $r=1.1$ ) the strong zero-frequency spectral component indicates that the field is asymmetric about the origin. A set of heterodyne spectra is shown in Fig. 7 for various degrees of detuning in a laser having a threshold parameter of $r=1.4$. These spectra correspond to the time-domain intensity plots given in Fig. 1. It is evident from both the time-domain waveforms and the heterodyne spectra that the pulsations are irregular for slight detunings and nearly periodic for larger detunings.

\section{DISCUSSION}

Laser instabilities often reveal themselves as rapid timedependent intensity fluctuations in cw-excited laser oscillators, and direct time-domain measurements of these fluctuations can be a convenient approach to the study of such instabilities. On the other hand, there may be important reasons for also studying the frequency-domain spectra associated with the pulsations. When the instantaneous frequency is measured, it becomes possible to determine the actual field amplitude rather than the intensity. Furthermore, the loss of information associated with homodyne and heterodyne spectral measurements may be more than compensated for by the opportunity to use powerful noise-reduction methods, such as synchronous detection. These methods may be necessary if one is to extract useful information from the weak signals that are sometimes characteristic of the most interesting operating regions. Also, spectral measurements can often provide a more direct indication of transitions to chaos than is possible from time-domain pulsation sequences. This study emphasized the various types of frequency information that can be extracted from a standing-wave model for a low-pressure xenon laser. The same model and methods should be applicable to a wide range of laser types, and it is only necessary to introduce into the model the appropriate parameter values for a laser of interest. 


\section{ACKNOWLEDGMENTS}

The author is grateful for valuable discussions with N. B. Abrahàm and D. L. MacFarlane. I also note with sadness the recent passing of A. T. Forrester and acknowledge his profound contributions to the development of optical homodyne and heterodyne detection methods and especially his personal interest in my own studies. This research was supported in part by the National Science Foundation and by Tektronix, Inc.

\section{REFERENCES}

1. L. W. Casperson, J. Opt. Soc. Am. B 5, 958 (1988).

2. L. W. Casperson and A. Yariv, IEEE J. Quantum Electron. QE8, 69 (1972).
3. See, for example, N. B. Abraham, T. Chyba, M. Coleman, R. S. Gioggia, N. J. Halas, L. M. Hoffer, S. N. Liu, M. Maeda, and J. C. Wesson, in Third New Zealand Symposium on Laser Physics, J. D. Harvey and D. F. Walls, eds., Vol. 182 of Springer Lecture Notes in Physics (Springer-Verlag, Berlin, 1983), p.
107.

4. J. H. Eberly and K. Wodkiewicz, J. Opt. Soc. Am. 67, 1252 (1977).

5. J. D. Cresser, Phys. Rep. 94, 47 (1983).

6. L. W. Casperson and A. Yariv, Appl. Phys. Lett. 17, 259 (1970).

7. A. T. Forrester, T. A. Gudmundsen, and P. O. Johnson, Phys. Rev. 99, 1691 (1955).

8. R. H. Brown and R. Q. Twiss, Nature 177, 27 (1956).

9. A. T. Forrester, J. Opt. Soc. Am. 51, 253 (1961).

10. L. W. Casperson, J. Opt. Soc. Am. B 2, 73 (1985). 